\title{
Imaging and Clinical Findings in Patients with Aberrant Course of the Cervical Internal Carotid Arteries
}

\author{
Alberto Muñoz ${ }^{1} *$, Joaquín De Vergas ${ }^{2}$ and José Crespo ${ }^{3}$ \\ ${ }^{I}$ Dpto. de Radiología, Facultad de Medicina, Ciudad Universitaria, Pabellón II, Planta 1ª , 28040, Madrid, Spain \\ ${ }^{2}$ Servicio de ORL, Hospital Universitario "12 de Octubre”, C/ Glorieta de Málaga, 28041, Madrid, Spain \\ ${ }^{3}$ GIB - LIA, DLSIIS, Facultad de Informática, Universidad Politécnica de Madrid, 28660 Boadilla del Monte (Madrid), \\ Spain
}

\begin{abstract}
Background and Purpose: Aberrrant course of the cervical internal carotid arteries (ICAs) may result in submucosal masses in the posterior pharyngeal wall, may cause confusion at physical examination, may be symptomatic, and can be at risk of surgical injury. The aim of this report is to present the clinical and imaging characteristics associated with aberrant course of the cervical portion of the ICAs.

Methods: Imaging studies of 5.500 patients were prospectively selected from CT studies of the head and neck performed in a five years period, in which the course of the one or both ICAs at the level of the hypopharynx and oropharynx was assessed as aberrant by means of a proposed classification. We then reviewed the medical records to establish which symptoms were present and if these symptoms could be caused by these variations in the course of the ICAs. In selected cases, further studies including magnetic resonance (MR) imaging, MR angiography (MRA), or selective catheter angiography were obtained.

Results: In our restricted classification, we found $14(0.2 \%)$ patients who met the cervical ICA aberrancy criteria. In all patients contrast enhanced CT or CT angiography was performed, 4 also have had MRI and MRA, and in two additional catheter angiograms were performed. Mean age was 62 years. Eight patients were male and seven were female. Four patients $(28 \%)$ were considered to have clinical symptoms related to aberrant course of the ICAs. In most of the symptomatic patients both ICAs had aberrant courses. Overall, the course of the right ICA was aberrant in $43 \%$, the left ICA in $14 \%$, and both in $43 \%$. In $50 \%$ of the cases the aberrancy of the artery was focal (localized to the oropharynx or laryngopharynx) and in the other $50 \%$ it involved the entire cervical course of the ICA.

Conclusions: In most of our patients variations in the course of the cervical portion of the ICA involved the right side and were asymptomatic, except with regard to potential surgical risks. However, in about $25 \%$ of our patients these variations were thought to be the culprit of patient complaints, particularly oropharyngeal pulsatile sensation. Furthermore, extreme degrees of medialization of the ICAs resulted in progressive symptoms including hoarseness and upper respiratory distress.
\end{abstract}

Keywords: Internal carotid artery, anomalies, internal carotid artery, aberrancy, head and neck, carotid artery.

\section{INTRODUCTION}

Medialization, tortuosity, elongation, ectasia or aberrancy of the carotid arteries are interchangeable terms frequently noted when performing angiography of the neck $[1,2]$, and was first classified by Metz et al. by its angiographically appearance [3]. Most variations in the course of the common carotid artery have no clinical significance. Extreme tortuosity (kinking and coiling) in the proximal portion of an internal carotid artery (ICA) was associated with an increased risk of dissection, stroke and injury at surgery $[1,3]$. Medialization of the mid portion of the cervical ICA at the level of the oropharynx has been addressed in the otolaryngologic literature primarily in reference to the risks of carotid injury during surgery $[4,5]$ and only sporadically in the radiologic

*Address correspondence to this author at the Dpto. de Radiología, Facultad de Medicina, Ciudad Universitaria, Pabellón II, Planta 1a, 28040 , Madrid, Spain; Tel: 91-3941514; Fax: (34) 91 3941514;

E-mail: almugo7557@gmail.com literature [6-9]. These reports were focused on the potential damage to the cervical ICA in the setting of minor surgical procedures such as tonsillectomy or adenoidectomy, and deep biopsies. Also, these anomalies can also be of importance as they may cause compressive symptoms. However, these questions have scarcely been discussed.

The purpose of this report is to review the imaging features and clinical significance in variations in the course of the ICA, particularly at the level of the hypo and oropharynx (cervical portion), which would correspond angiographically with the sternomastoid and/or retrostyloid portions of the cervical inernal carotid artery [9] and to propose a restricted classification of the cervical ICA aberrancy, based on modern crossed-sectional computerized imaging.

\section{METHODS}

We prospectively study the cross sectional imaging of CT studies of the head and neck of patients referred to a single referral institution in a five years period of time. Most 
patients were referred from ENT and maxillofacial services for a wide range of symptoms, most of them non-specific. About 5.500 examinations were reviewed. We then identify 14 patients whose cervical portion of one or both ICAs was defined as aberrant (see below). We also reviewed the charts of these patients and correlated the clinical symptoms with the findings on the imaging studies. All patients had contrast-enhanced CT of the neck (using 3 to $5 \mathrm{~mm}$ thickness slices). In selected four cases, particularly in patients with hoarseness or respiratory distress, further MR imaging (MRI) studies were performed, using axial $4 \mathrm{~mm} \mathrm{T1}$ and T2 weighted images, post contrast T1weighted images, and MR angiography (MRA). Finally, in 2 patients selective catheter digital substration angiography of the neck arteries was performed.

We propose a new restricted classification of cervical ICA aberrations, based on current cross-sectional computerized imaging tools that allow radiologists to determine actual aberrancy from former angiographically variations in shape or trajects, because these new methods allows us to distinguish precisely the vascular and perivascular anatomical landmarks, in particular in relation with the boundaries of head and neck spaces.

We consider the cervical ICA to have an aberrant course on cross sectional imaging when the vessel meet the following anatomic criteria: 1- Identification of the vessel immediately adjacent to the deglutory component of the hypopharynx and/or oropharynx (submucosal traject); 2- Indentation or bulging on the oropharyngeal or hypopharyngeal lumen by the artery; 3-Visualization of an abrupt horizontal change of the course of the vessel denoting its medialization (a finding that could be unilateral or bilateral); and 4- Retropharyngeal position. To all of the above categories we assigned then a grade 1 if the aberrant course did not reach the midline and a grade 2 in those patients whose arteries reached the midline (one or both). We further divided the patients into 3 groups. Group A included those cases in whom the displacement or bulging involved the oropharynx; group B included those cases in whom the involvement affected the hypopharynx or laryngopharynx, and group $\mathrm{C}$ included those cases with generalized involvement.

\section{RESULTS}

Table 1 shows the clinical and imaging data of our patients. Fifty three percent of the patients were male and $47 \%$ female. Mean age was 62 years. The incidence of aberrancy involving the cervical portion of ICAs, whether unilateral or bilateral, was $0.2 \%$.

The right cervical ICA had an aberrant course in six patients (43\%), the left in two (14\%) and both arteries (kissing carotids) were involved in six (43\%) patients (Fig. 1). In $50 \%$ of patients the aberrant course was focal (oro or hypopharynx) while in the other $50 \%$ it was generalized. In 10 patients the aberrant coursing vessel caused radiological evident bulging in the oropharyngeal wall (75\%), (Fig. 2). In four patients $(28 \%)$ one of the arteries was found in a retropharyngeal position. In two of these patients (14\%), both internal jugular veins had also a medialized course paralleling the course of ICAs.
In four patients $(28 \%)$ the presenting symptoms that prompted the imaging study were thought to be related to the aberrant course of the arteries.

Symptoms included pulsatile masses, longstanding hoarseness, foreign body sensation, and upper respiratory distress. In 6 out of 14 patients (42\%) oropharyngoscopy revealed a retropharyngeal submucosal bulging at the site of the aberrant course and on physical examination pulsation was palpated in four of them (28\%). Vascular risk factors such as arterial hypertension, diabetes mellitus, and hypercholesterolemia were present in $28 \%$ of patients.

According to our proposed classification, four patients were considered category A, 1; one category A, 2; one category $\mathrm{B}, 1$; six category $\mathrm{C}, 1$; and two category $\mathrm{C}, 2$. Patients included into category $\mathrm{C}$ and/ or grade 2 were the most likely to be symptomatic (Figs. 3 and $\mathbf{4}$ ).

\section{DISCUSSION}

Aberrant course of one or both ICAs, ICA transposition, ectasia, or tortuous ICAs have been interchangeable clinical terms that have been used to describe an abnormal course of the ICAs and although the classification of Metz et al. [3] is sufficient to outline the anatomical course of the ICA, to illustrate the vessel's shape, and to evaluate whether symptoms of cerebrovascular insufficiency may derive from an ICA variation, this classification does not consider the vessel's relation to the pharyngeal wall and is therefore clinically insufficient to assess the risk potential of iatrogenic or accident-caused ICA injury, as stated by Pfeiffer and Reeder [10], or potential clinical symptoms related to vascular compression.

To date, no consistent radiological criteria on crosssectional imaging has been made, and particularly to link this morphologic variations with potential vascular compressive symptoms. Furthermore, the significance of these anomalies varies with the segment of the artery that is involved. Although we focused on the anomalies of the cervical portion of the ICA, other publications have reviewed the anomalies along the entire course of the extracranial ICAs that have no role for helping ENT surgeons to avoid surgical risk of arterial lesions [10], or explaining symptoms potentially related to vascular compression, which is one of the purposes of our study.

We believe that our proposed new restricted classification of cervical ICA aberrations, based on current crosssectional computerized imaging tools, allows radiologist to determine actual cervical ICA aberrancy precisely and distinguish it from former angiogrphically variations in shape or trajects because the unique anatomic landmarks that these methods can display, in particular when we consider the vascular space with other adjacent head and neck spaces [11-13].

For a full understanding of the ICA aberrancy, a brief anatomic and embryologic review is necessary. The right common carotid artery usually arises from the division of the innominate trunk, whereas the left one usually arises directly from the aortic arch. Both common carotid arteries typically bifurcate at the C3-C4 level and extend vertically to the orifice of the carotid canal at the base of skull. The segment from the bifurcation to the skull base is called the cervical or ascending segment. The cervical segment of an ICA is further divided into a lower 'sternomastoid portion' and an upper 
Table 1. Clinical and Imaging Findings in 14 Patients with Aberrant Course of Cervical Internal Carotid Arteries

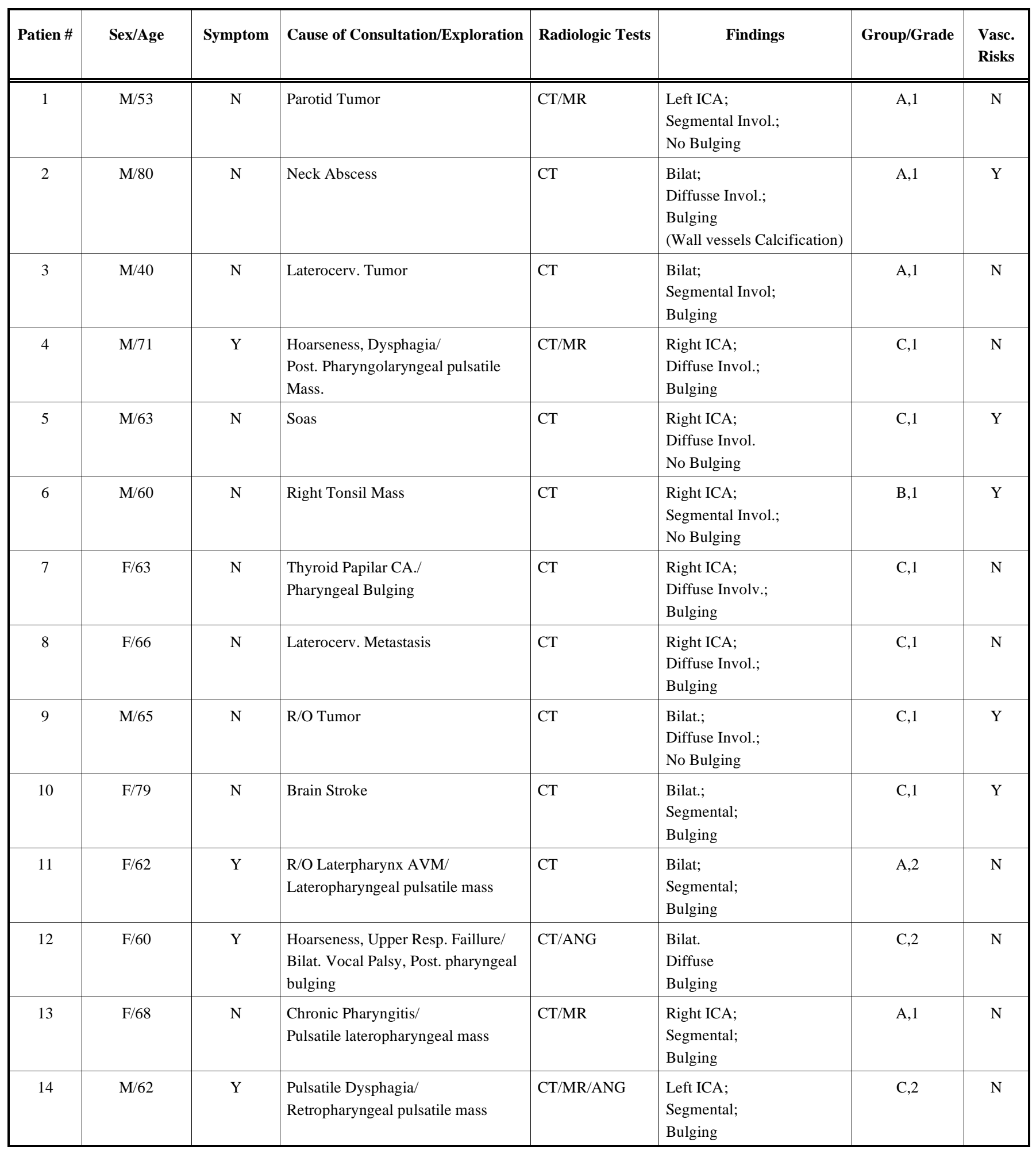

ANG: Angiography

AVM: Arteriovenous malformation

CA: Carcinoma

Invol: Involvement

F: Female

M: Male

$\mathrm{N} ; \mathrm{No}$

Vasc: Vascular

SOAS: Sleep obstructive apnea syndrome

Y: Yes 


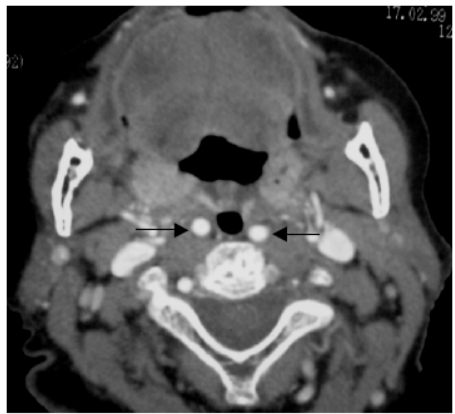

$\mathbf{A}$

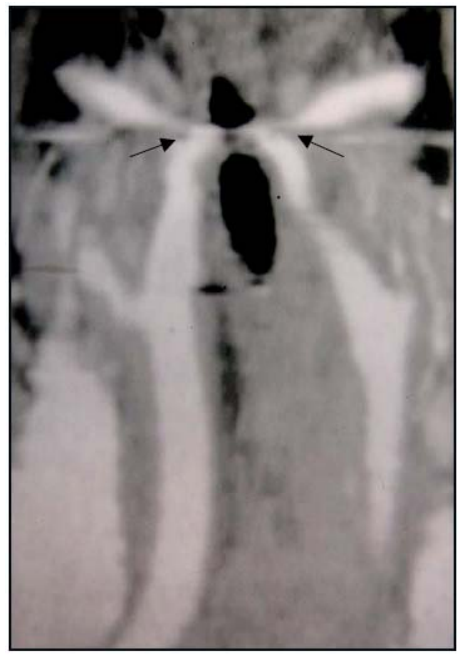

B



C

Fig. (1). (A-C). Bilateral medialization of the cervical course of both internal carotid arteries (patient \#10). Enhanced helical CT scan throughout the neck.

A: Axial image through the oropharynx shows marked medialization of both ICA's with no focal lumen bulging on the oropharyngeal lumen (arrows).

B and C: Maximum Intensity Projection (MIP) (B) and Surface Shade Reconstruction (C) from axial source images show medialization of both ICA's with abrupt change in the course of both arteries at the level of the oropharynx (arrow). After a short horizontal traject the arteries regain their normal position before reaching the base of the skull.

(Radiologic classification of this patient was considered C,1).

'retrostyloid or parapharyngeal portion [9], and in most individuals is lateral to the pharyngeal wall.
The ICA courses within the carotid sheath, accompanied by the internal jugular vein and vagus nerve within the vascular space. The suprahyoid portion of the carotid sheath does not have complete anatomic boundaries, either because there is no true fascial sheath or because the sheath is incomplete. Thus, it may potentially communicate with the parapharyngeal or retropharyngeal spaces [11-15].

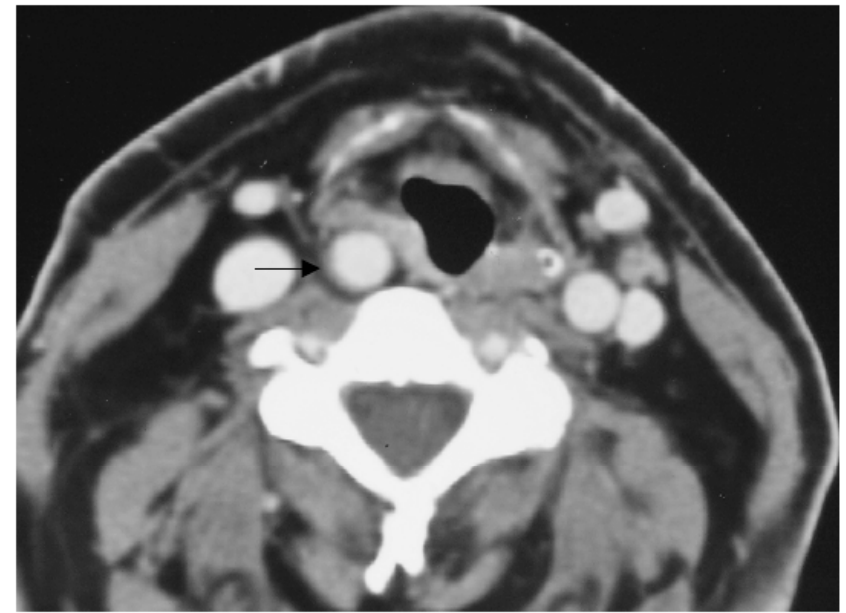

Fig. (2). Right aberrant cervical internal carotid artery promotes focal pharyngeal bulging (patient \#4).

Axial enhanced CT scan t throughout the supraglottic level shows aberrant right ICA causing focal bulging at the level of the right pyriform sinus with right aryepiglottic fold displacement (arrow). (Radiological classification of this patient was considered C,1).

Two different clinical and radiological entities related to aberrancy or morphological variations of the extracranial ICA course are recognized. The first involves the common carotid artery and the second the cervical portion of the ICA. The common carotid arteries are anchored distally by fascial attachments and by the branches of the external carotid arteries. In addition, on the right side the common carotid artery is relatively fixed at its origin from the innominate artery. With aging and other acquired factors such hypertension, the aortic arch elongates and the right common carotid artery is displaced cephalic [16-20]. As a consequence, the artery buckles to accommodate the decreased distance between its proximal and distal ends [19, 21]. Furthermore, the right common carotid artery is subjected to a greater systolic impulse that furthers accentuates the mechanical consequences of the elevation of the aortic arch. These factors are less prominent on the left side since the left common carotid artery arises directly from the middle aortic arch (and not the ascending as the right does) and has a longer course. Therefore, any buckling that occurs is distributed over a greater distance, thereby minimizing the occurrence of focal tortuosity $[2,22]$. But additional embryologic mechanisms have also been proposed to explain the changes in shape of ICA. Embryological, the ICAs are formed from the third aortic arch and from the dorsal aorta. In the embryo, the ICAs are normally kinked or coiled. As the heart recedes into the thorax, the ICAs are stretched and their kinks are eliminated [3, 23]. Failure of descent of the heart and incomplete uncoiling the ICAs is thought to be the principal cause of vascular loops in this region, whether by its embryological persistence or by facilitating the appearance of kinking over the 


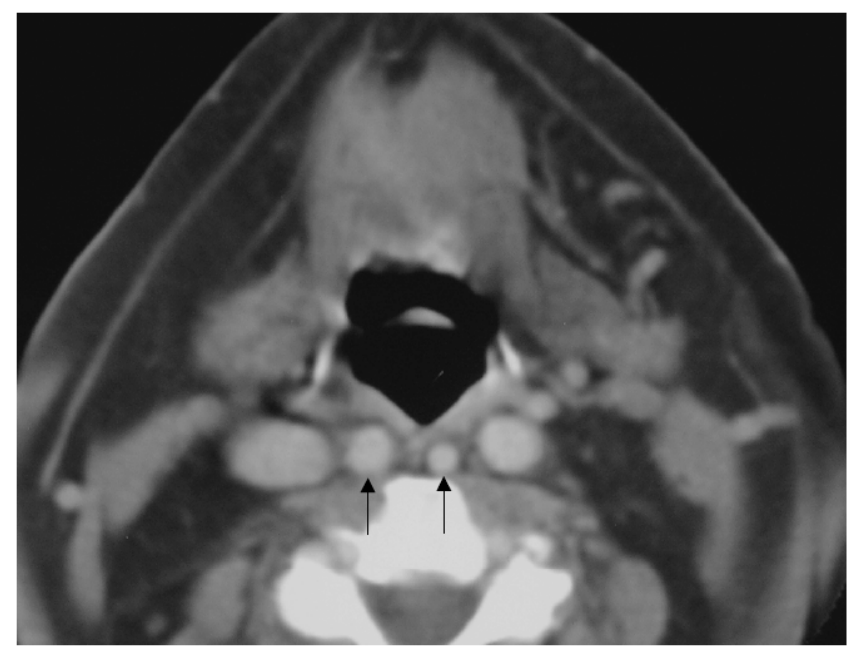

A

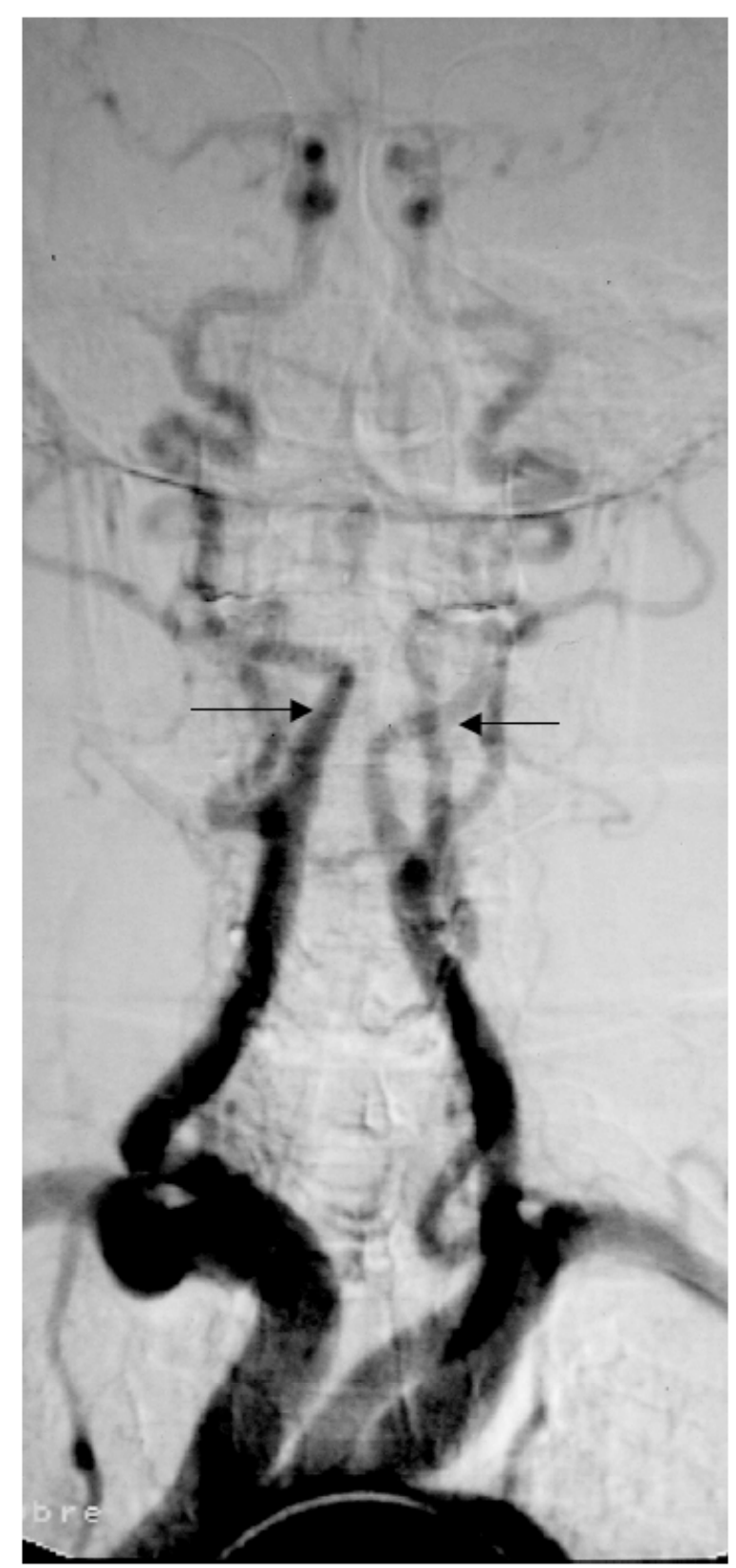

B
Fig. (3). contd.....

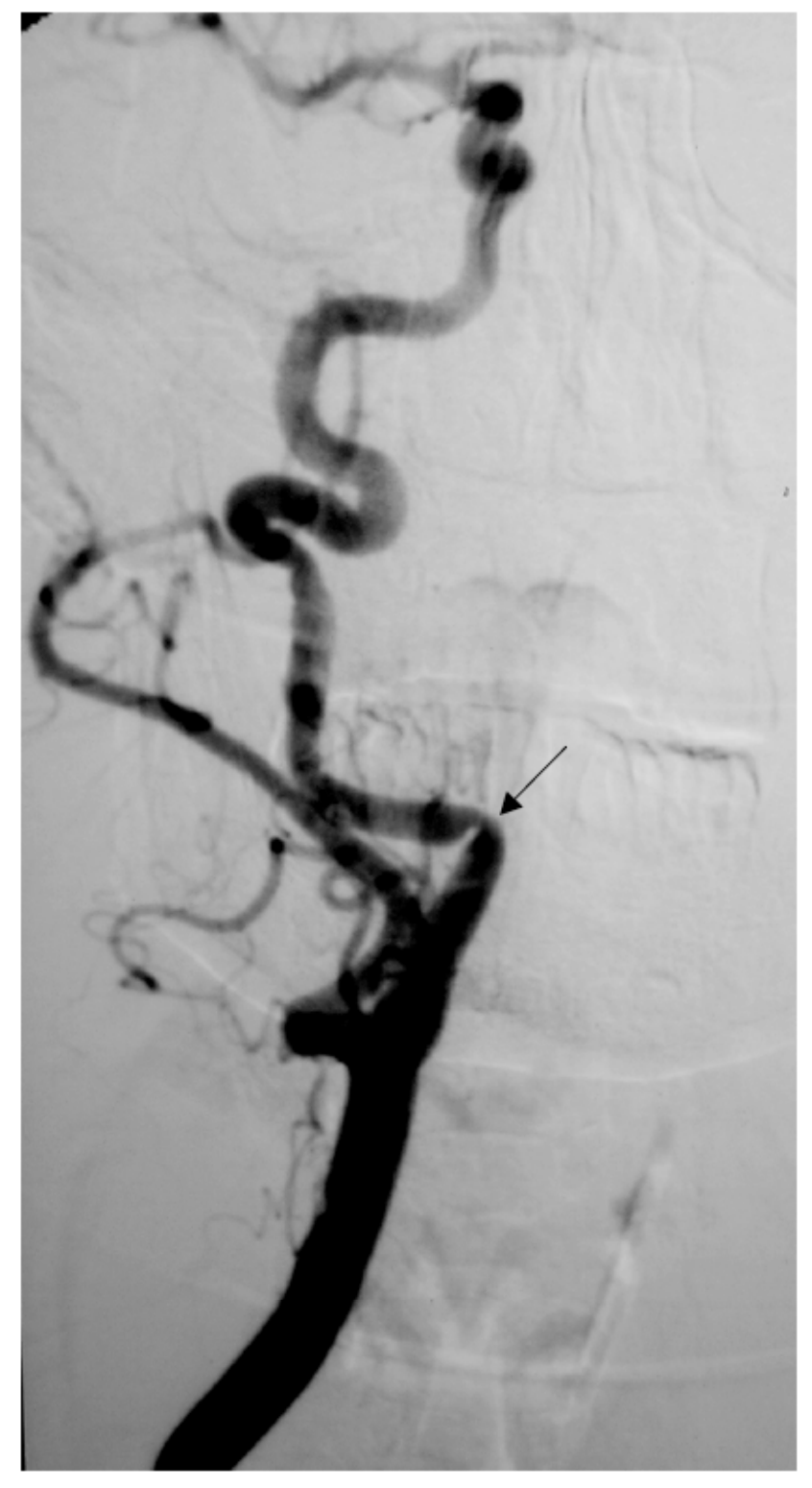

C

Fig. (3). (A-C). Extended bilateral retropharyngeal course of both internal carotid arteries (kissing carotids), (patient \#12).

A: Axial contrast CT scan in early-enhanced phase throughout the oropharynx. Notice medialization of both ICA's reaching the retropharyngeal midline (arrows), and also medialization of both internal jugular veins.

B and C: Digital subtraction angiography in anteroposterior projection.

Upper aortogram of both ICA's in the early arteriographic phase (B) shows bilateral medialization of the cervical segment of the ICA's (arrows). Notice tortuosity of the proximal portions of the right common and external carotid arteries. Selective injection of the right common carotid artery in early arteriographic phase (C) shows progressive medialization of the proximal segment of ICA and then abrupt change in the course of the artery and then the artery regains its normal position after a short horizontal segment. Notice extrinsic arterial notch pinpointing the place of arterial course change and producing a focal non-atherosclerotic stenosis (arrow).

(Radiological classification of this patient was considered C,2.) 
A

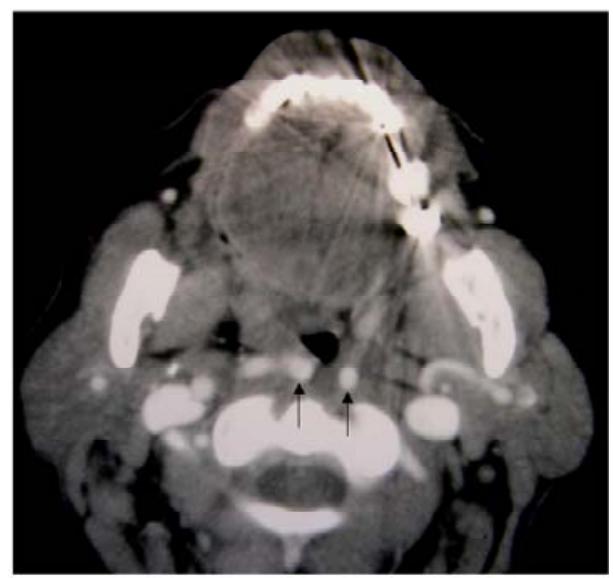

B

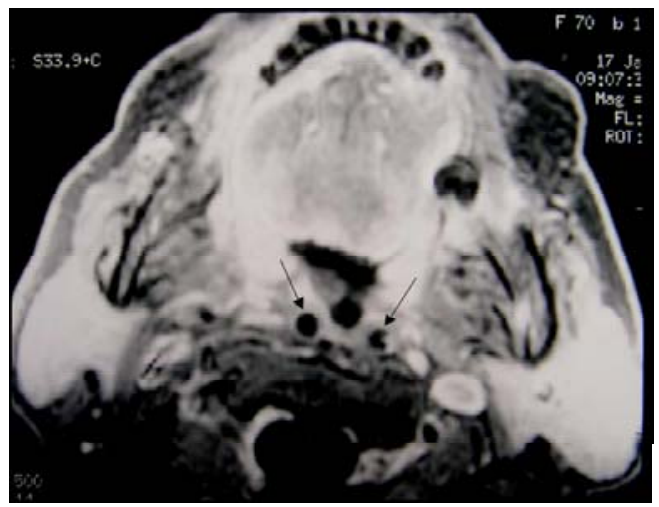

C

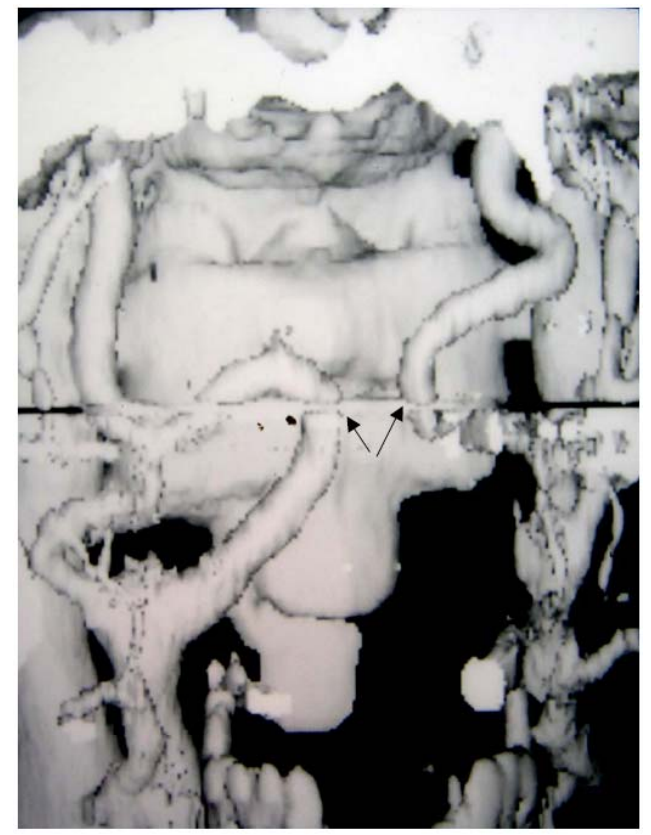

Fig. (4A-C). Focal bilateral course of both internal carotid arteries at the level of the oropharynx (patient \# 11).

A and B: Axial enhanced CT and enhanced T1-weighted (600/12; TR/TE) MR scans through the oropharynx shows bilateral medialization of the course of both ICA's (kissing carotids), running in the retropharyngeal space.

C: Maximum Intensity Projection (MIP) from axial sources images of CT and MR show medialization of both ICA's, with abrupt change in the course of both arteries at the level of the oropharynx and, thereafter, upper regaining its normal course (arrows).

(Radiological classification of this patient was considered A,2). years in the presence of vascular risk factors [3, 24]. Hence, the most common site of ICA coiling in the adult corresponds to the anteromedial bend of the embryonic vessel coil just on the level of the tonsillar fossa. Therefore, the term "dangerous loop" of the ICA has been coined $[5,10]$.

Alternatively, or in a complementary fashion, it seems that elastin deficit of the arterial wall, whether congenital or acquired due to risk vascular factors and aging, promotes arterial wall weakness and can lead to redundancy, dilatation, ectasia, kinking, coiling, or aneurysmal dilatation of the ICAs [25-29]. Lately, it is generally believed that coiling is due to embryological causes while tortuosity and kinking are associated with fibromuscular dysplasia, atherosclerosis, or other acquired factors $[20,30]$

Although the relationship between the morphologic variations of the ICAs and the risk of stroke is controversial, it seems that there is an association between extreme degrees of tortuosity (kinking and coiling) and stroke by precipitating focal atherosclerotic plaque formation or by increasing susceptibility to dissection $[1,3,17,31-38]$. These major angulations tend to occur near the origin of the ICA (sternomastoid portion of the cervical portion of ICA) or in its distal portion at the level of the atlas and axis [16-33].

Variations in the shape and position of the middle segment of the cervical ICA, known as "aberrant carotid artery" have been addressed to some extent in the otolaryngologic literature $[4,5,10,29]$, and only anecdotally reported in the radiological literature [6-8], but no consistent criteria on cross-sectional advanced computerized imaging have been defined. Surgical interest in this abnormality stems from the risk of exanguinating hemorrhage during tumor resections and other procedures such as tonsillectomy, adenoidectomy, or oropharyngoplasty [5]. Morbidity of hemorrhage sufficient to require transfusion in the setting of tonsillectomy and adenoidectomy occurs in about $1 / 2,400-2,500$ cases [5, 39], particularly related to bleeding from the rich arterial supply to this region of the pharynx, including ascending pharyngeal, lingual, facial, and internal maxillary branches of the ECA. However, it is unknown the incidence of hemorrhage due to aberrancy of the cervical portion of the ICA.

Clinically, $1-16 \%$ of the population has detectable lateral pharyngeal wall pulsations, depending of the individual phenotype with a slight female predominance $[5,10]$. In about $70 \%$ of the patients these pulsations are unilateral and mostly on the right side (67\%). In $30 \%$ of patients there are bilateral involvement. Midline posterior interposition of both cervical ICAs is very rare with an incidence of $2.6 \%$ in patients with pharyngeal wall pulsations [4].

On the other hand, as mentioned earlier, the carotid sheath may not have well-defined boundaries, especially in its upper region. Therefore, the structures contained within the sheath, particularly the ICA but also the jugular vein as seen in some of our patients, can potentially course in the retropharyngeal space. This extreme deviation, in which an ICA is located between the pharynx and the spine, has been rarely reported in the literature [40].

The incidence of aberrancy of ICAs defined by the above criteria in our population was $0.2 \%$. This incidence is far below compared to the historical clinical series focused on the entire ICA, and should not be extrapolate with those se- 
ries since in this report we have proposed an anatomic and radiologic criteria useful particularly on cross sectional imaging and specifically addressed for the cervical portion of the ICA that takes in consideration not only the anatomic course o the ICA but also its relation with the adjacent head and neck cervical spaces.

In a previous anatomic work, Paulsen et al. [41] analyzed the variations in shape of ICA in 164 head and neck preparations from adult cadavers. They classified the course of the ICA according to its curvature from the vertical axis. From a total of 282 dissected carotid sheets, they found no curvature in $67.7 \% ; 26,2 \%$ had medial, lateral, or ventrocaudal curve; $4,2 \%$ showed kinking; and $1.7 \%$ showed coiling. They concluded that whereas coiling was related with aging, kinking was exacerbated by arteriosclerosis or fibromuscular dysplasia with advancing age and may be of significance in relation to the occurrence of cerebrovascular symptoms. However, this work is only anatomic and taken from cadaveric specimens. No clinical or radiological correlation was attempted.

Recently, Pfeiffer and Ridder [10] have propose a clinical and radiological grading system for parapharyngeal ICA aberrations based on its location and the minimum distance to the pharyngeal's wall that was found to have significant impact on the risk potential for surgical ICA injury. The authors did neither mention the incidence of these anomalies in their study, nor did they explain the relationship between clinical symptoms and cervical ICA aberrancy.

Also, they concluded that the anatomic classification of the vessel's course, according to Weibel and Fields [42], in tortuosity, kinking, and coiling did not correlate with the vessel's minimum distance to the pharyngeal wall or the malformation's pharyngeal level.

In our population the percentage of symptomatic patients is low $(0.2 \%)$ and it includes a subset of patients with aberrant cervical ICA that are symptomatic for mass effect symptoms. The right cervical ICA was most commonly involved and both arteries were involved in $6(43 \%)$ patients and 4 patients (28\%) were symptomatic from this condition. The submucosal location of the vascular structures would contribute to the development of symptoms, such as oropharyngeal pulsatile sensation, foreign body sensation, and dysphagia experienced by some of our patients. However, chronic hoarseness and upper respiratory distress have not been specifically linked to aberrant ICA, although it has been previously recorded in the setting of aberrancy of the cervical internal carotid aberrancy [6] but could have been attributed to other anatomic and degenerative changes.

Again, anatomical and embryological reasons may explain this infrequent symptom. The superior laryngeal nerve, that gives-rise to a branch that supplies the motor fibers of the cricothyroid muscle passes caudal and medial to the cervical portion of ICA [43]. Thus, the nerve is at risk of stretching and damage in cases of extreme ICA displacement (similar to what occurs to the left recurrent laryngeal nerve when the left atrium enlarges). This may explain the longstanding hoarseness that two of our patients experienced, that could have not been attributed to other factors after a thorough clinical and endoscopic examination. In particular, one of our patients (\#12) with chronic hoarseness for over 20 years presented with progressive respiratory distress and posterior oropharyngeal bulging. Imaging studies demonstrated striking bilateral medialization of both ICAs at the level of the hypo and oropharynx (Fig. 3). This patient is a representative example of an extreme degree of medialization of both cervical ICAs likely due to both congenital medialization of this segment of the ICA and additional acquired factors, such as age, resulting in hoarseness and final upper respiratory failure as a consequence of posterior airway compression. This example would also support the hypothesized embryological basis of the ICA medialization, which would correspond to the anteromedial bend where the ninth cranial nerve crosses the ICA, first postulated by Kelly [4]. Our radiological studies, including both helical CT reconstructions (Fig. 1) and angiography (Fig. 2), show abrupt change in the course of the ICA at the level of the oropharynx, with extrinsic indentation at the cervical ICA promoting non-atherosclerotic stenosis. This finding would also suggest a specific anatomic lieu where this embryological bend took place, although we have no surgical confirmation.

Nevertheless, most patients who develop symptoms are only symptomatic of pulsatile retropharyngeal mass, most of them in the right side, although in our report one patient with these characteristics were found in the left side.

Finally, of interest is to note that in two patients who exhibited extreme medialization of the cervical ICA, the jugular vein was also medialized (Fig. 3). This finding could also be interpreted in the light of the variations of the carotid space, as a result of its incomplete anatomic boundaries in the middle and upper region of the neck.

\section{CONCLUSIONS}

We have presented a group of patients with aberrancy in the location and course of the middle cervical portion of the ICAs. In general, these variations involved the right side, were asymptomatic and are important to recognize to avoid surgical injury. In about one fourth of our patients these variations were thought to be the cause of patient's complaints, particularly oropharyngeal pulsatile sensation. In rare instances, extreme degrees of medialization, particularly when bilateral, may result in progressive symptoms including hoarseness and upper respiratory distress. These variations in the course of the ICA have an embryological basis, but are also probably facilitated by the absence of complete anatomic boundaries of the carotid sheath, and by acquired factors, such as aging and hypertension.

\section{ACKNOWLEDGEMENT}

This work has been supported in part by "Ministerio de Ciencia e Innovación" of Spain (Ref.: TIN2007-61768).

\section{REFERENCES}

[1] Leipzig TJ, Dohrmann GJ. The tortuous or kinked carotid artery: pathogenesis and clinical considerations. Surg Neurol 1986; 25: 478-86.

[2] Godin MS, Rice JC, Kerstein MD. Tortuosity of the right common carotid artery simulating aneurysm. South Med J 1988; 81: 138285

[3] Metz H, Murray-Leslie RM, Bannister RG, Bull JWD, Marshall J. Kinking of the internal carotid artery in relation to cerebrovascular disease. Lancet 1961; 1: 1424-26.

[4] Kelly AB.Tortuosity of the internal carotid artery in relation with the pharynx. J Laryngol Otol 1925; 40: 15-23.

[5] Ricciardelli E, Hillel AD, Schwartz AN. Aberrant carotid artery Arch Otolaryngol Head Neck Surg 1989; 11: 5519-22. 
[6] Shanley DJ. Bilateral aberrant cervical internal carotid arteries Neuroradiology 1992; 35: 55-6.

[7] Fix TJ, Daffner RH, Deeb ZL.Carotid transposition: another cause of wide retropharyngeal soft tissue. Am J Roentgenol 1996; 167: 1305-7.

[8] Vega J, Gervas C, Vega-Hazas G, Barrera C, Biurun C. Internal carotid artery transposition: another cause of widening of the retropharyngeal space. Eur Radiol 1999; 9: 347-8.

[9] Newton T, Potts D, Eds. Radiology of the skull and brain. Angiography. Mosby, St. Louis 1974; pp. 1202-7.

[10] Pfeiffer J, Ridder GJ. A clinical classification system for aberrant internal carotid arteries. Laryngoscope 2008; 118: 1931-6.

[11] Gerodinsky M, Holyokc EA. The fascial and fascial spaces of the head and neck. Am J Anat 1938; 63: 367-408.

[12] Smoker WRK. Normal anatomy of the neck. In: Som PM, Curtin HD, Eds. Head and Neck imaging. Mosby Yearbook Inc. 1996; pp. 711-37.

[13] Som PM, Curtin HD. Fasciae and Spaces. In: Som PM, Curtin HD, Eds. Head and Neck imaging. Mosby Yearbook Inc. 1996; pp. 73847.

[14] Grodinsky M, Holyoke EA. The fascial and fascial spaces of the head, neck and adjacent regions. Am J Anat 1938; 63: 367-408.

[15] Rouviere H. Anatomie Humaine. Tome I. Paris: Masson et Cie, 1959; pp. 469-544.

[16] Bauer R, Sheehan S, Meyer JS. Arteriographic study of cerebrovascular diseases: II. Cerebral symptoms related due to kinking, tortuosity and compression of carotid and vertebral carotid arteries in the neck. Arch Neurol 1961; 4: 119-31.

[17] Perdue GD, Barreca JP, Smith RB, King OW. The significance of elongation and angulation of carotid artery: a negative view Surgery $1975 ; 77: 45-52$.

[18] Lentino W, Principato DJ, and Poppel MH. Buckling of the carotid artery demonstrated by angiocardiography Ann Intern Med 1956; 44: 103-1007.

[19] Cocchrane J. The S-shaped carotid in Africans. S Afr Med J 1948; 22: 230-3.

[20] Stone PW, Lord JW Jr. Pseudoaneurysm of right common carotid artery. NY State. Med J 1960; 60: 1239-41.

[21] Parkinson J, Bedford ED, Aldmond S. The kinked carotid artery that simulates aneurysm. Br Heart J 1939; 1: 345-61.

[22] Deterling RA, Jr. Tortuous right common carotid artery simulating aneurysm. Angiology 1952; 3; 383-491.

[23] Skillern PG. Anomalous internal carotid artery and its clinical significance in operations in tonsils. JAMA 1913; 60: 172-3.

[24] Mukherjee D, Inahara T. Management of the tortuous internal carotid artery. Am J Surg 1985; 149: 651-5.

[25] Dobrin PB, Schwarcz TH, Baker WH. Mechanism of arterial and aneurysmal tortuosity. Surgery 1988; 104: 568-71.
[26] Oschner JL, Hughes JP, Leonard GL, et al. Elastic tissue dysplasia of the internal carotid artery. Ann Surg 1977; 185: 684-91.

[27] Dobrin PB, Baker WH, Gley WC. Ellastic and collagenolytic studies of arteries: implications for mechanical properties of aneurysm. Arch Surg 1984; 119: 405-9.

[28] Muñoz A, Campollo J, Vergas J. Bilateral internal carotid aneurysms presenting as a nonpulsatile parapharyngeal mass: complementary diagnosis by CT, MR Imaging, and digital subtraction angiography. Am J Neurorad (AJNR) 2001; 22: 864-6.

[29] Ozgur Z, Celik S, Govsa F, et al. A study of the course of the internal carotid artery in the parapharyngeal space and its clinical importance Eur Arch Otorhinolaryngol 2007; 264: 1483-9.

[30] Schenk P, Temmel A, Trattnig S, Kainberger F. Current aspects in diagnosis and therapy of carotid artery kinking. HNO 1996; 44: 178-85.

[31] Boucher A. Megadolicho-arteries of the carotid system. Apropos of a case operated with success. Rev Otoneuroophtalmol 1987; 50: 95-8.

[32] Derrick JR. Carotid kinking and cerebral insufficiency. Geriatrics 1963; 18: 272-5.

[33] Grass HH. Kinks and coils of the cervical carotid artery. Surg Forum 1959; 9: 721-4.

[34] Grant DN. Neurological manifestations associated with the internal carotid artery. Dev Med Child Neurol 1970; 12: 797-9.

[35] Stanton PE Jr, McClusky DA Jr, Lamis PA. Hemodynamic assessment and surgical correction of kinking of the internal carotid artery. Surgery 1978; 84: 793-802.

[36] Vannix RS, Jorgenson EJ, and Carter R. Kinking of the internal carotid artery. Clinical significance and surgical management. Am J Surg 1977; 143: 82-9.

[37] Vollmar J, Nadjafi AS, Stalker CG. Surgical treatment of kinked internal carotid arteries. Br J Surg 1976; 63: 847-50.

[38] Barbour PJ, Castaldo JE, Rae-Grant A, et al. Internal Carotid artery redundancy is significantly associated with dissection. Stroke 1994; 25: 1201-6.

[39] Pratt LW. Tonsillectomy and adenoidectomy: mortality and morbidity. Trans Am Acad Ophtalmol Otolaryngol 1970; 74: 1146-54.

[40] Curtin HD. The Larynx. In: Som PM and Curtin HD (Eds). Head and Neck imaging. Mosby Yearbook Inc., 1996; p. 612-707.

[41] Paulsen F, Tillman B, Christoforodis C, et al. Curving and looping of the internal carotid artery in relation to the pharynx: frecuency, embryology and clinical implications. . J Anat 2000; 197: 373-381

[42] Weibel J, Fields WS. Tortuosity, coiling, and kinking of the internal carotid artery. I. Etiology and radiographic anatomy. Neurology 1965; 15: 7-18.

[43] Gray H. Gray’s anatomy. Philadelphia: Lea \& Febiger, 1966. 